\title{
Reduction of falls in a rat model of PD falls by the M1 PAM TAK-071
}

\author{
Aaron Kucinski ${ }^{1}$ - Martin Sarter ${ }^{1}$ (D) \\ Received: 27 October 2020 / Accepted: 8 March 2021 / Published online: 18 March 2021 \\ (C) The Author(s), under exclusive licence to Springer-Verlag GmbH Germany, part of Springer Nature 2021
}

\begin{abstract}
Rationale In addition to the disease-defining motor symptoms, patients with Parkinson's disease (PD) exhibit gait dysfunction, postural instability, and a propensity for falls. These dopamine (DA) replacement-resistant symptoms in part have been attributed to loss of basal forebrain (BF) cholinergic neurons and, in interaction with striatal dopamine (DA) loss, to the resulting disruption of the attentional control of balance and complex movements. Rats with dual cholinergic-DA losses ("DL rats") were previously demonstrated to model PD falls and associated impairments of gait and balance.

Objectives We previously found that the muscarinic M1-positive allosteric modulator (PAM) TAK-071 improved the attentional performance of rats with BF cholinergic losses. Here, we tested the hypotheses that TAK-071 reduces fall rates in DL rats.

Results Prior to DL surgery, female rats were trained to traverse a rotating straight rod as well as a rod with two zigzag segments. DL rats were refamiliarized with such traversals post-surgery and tested over 7 days on increasingly demanding testing conditions. TAK-071 (0.1, $0.3 \mathrm{mg} / \mathrm{kg}$, p.o.) was administered prior to daily test sessions over this 7-day period. As before, DL rats fell more frequently than sham-operated control rats. Treatment of DL rats with TAK-071 reduced falls from the rotating rod and the rotating zigzag rod, specifically when the angled part of the zigzag segment, upon entering, was at a steep, near vertical angle. Conclusions TAK-071 may benefit complex movement control, specifically in situations which disrupt the patterning of forward movement and require the interplay between cognitive and motor functions to modify movement based on information about the state of dynamic surfaces, balance, and gait.
\end{abstract}

Keywords PD falls $\cdot$ Acetylcholine $\cdot$ Dopamine $\cdot$ Basal forebrain $\cdot$ Cortex $\cdot$ Striatum $\cdot$ TAK-071

\section{Introduction}

In addition to the nigrostriatal dopamine (DA) deficiency and the associated efficacy of dopaminergic replacement therapy (DRT) to reduce motor symptoms, progressing Parkinson disease (PD) is characterized by neurodegeneration in non-DA systems and associated DRT nonresponsive features such as gait and balance disorder, postural instability, and cognitive impairments. Given the severe medical consequences of a resulting increase in the propensity for falls, investigating the pathophysiology underlying falls and the development of treatments to reduce fall risk are important objectives (Albin et al. 2018a; Weaver et al. 2016; Li et al. 2015; Paul et al. 2014; Cheng et al. 2014; Shine et al. 2013; Kehagia et al. 2013; Heremans et al. 2013; Amboni et al. 2013; Montero-

Martin Sarter

msarter@umich.edu

1 Department of Psychology, University of Michigan, 530 Church Street, Ann Arbor, MI 48103, USA
Odasso et al. 2012; Fasano et al. 2012; Cole et al. 2011; Taylor et al. 2009; Stack and Ashburn 2008; Langston 2006; Grimbergen et al. 2004).

Loss of cholinergic projections, which arise from the basal forebrain (BF) and innervate virtually all cortex as well as several thalamic nuclei, has been associated with increased falls in PD (Bohnen et al. 2019; Muller et al. 2013; Bohnen and Albin 2011; Bohnen et al. 2009; Rochester et al. 2012; Albin et al. 2018b). Cortical cholinergic signaling is necessary for the integration of exteroceptive and interoceptive cues into ongoing behavior, thereby permitting these cues to influence and, if previously associated with defined responses, control behavior (Sarter and Lustig 2020; Lu et al. 2020; Howe et al. 2017; Gritton et al. 2016; Berry et al. 2014; Howe et al. 2013; Parikh et al. 2007; Venkatesan et al. 2020; Picciotto et al. 2012). Therefore, PD falls have been extensively demonstrated to be closely associated with a decline in such attentional capacities (Stuart et al. 2017; Yarnall et al. 2011; Lord et al. 2010; Cools et al. 2010; Allcock et al. 2009; Yogev-Seligmann et al. 2008; Shumway-Cook and Woollacott 2000; Chen et al. 1996; Stam et al. 1993; Kim et al. 2017, 2019; Berry et al. 2017). 
We previously developed a rodent model of PD falls by demonstrating that rats with dual basal forebrain cholinergic and mediodorsal striatal DA losses ("DL rats") exhibit a heightened fall rate when required to traverse dynamic surfaces. A new behavioral set of tasks, called the Michigan Complex Movement Control Task (MCMCT), was shown to produce falls in DL rats that reflect the disruption of attentional-motor integration. Falls in DL rats were correlated with impairments in their sustained attention performance. Moreover, such falls were associated with behavioral risk factors comparable to those seen in PD fallers, including slower traversal speed, postural inefficiencies, and impairments in turning behavior. Correlations between cholinergic and dopaminergic losses and fall rates indicated that cholinergic losses unmask striatal deficits in the selection and sequencing of movements, particularly when movement corrections were triggered by paw misplacements or balancing errors (Avila et al. 2020; Koshy Cherian et al. 2019; Kucinski et al. 2017; Kucinski and Sarter 2016; Sarter et al. 2014; Kucinski et al. 2013). Thus, the performance of DL rats on the MCMCT specifically models falls resulting from a disruption of the brain's cognitive-motor interface.

The present experiment was designed to test the hypothesis that treating DL rats with a muscarinic M1 acetylcholine receptor (mAChR)-positive allosteric modulator (PAM) reduces their fall rate. The focus on M1 receptors as a key node in the attention-mediating functions of the BF cholinergic system in part arises from evidence showing that the cholinergic mediation of attention requires the M1-mediated generation of high-frequency oscillations in the cortex and of neuronal synchrony across multiple frequency bands (Howe et al. 2017; see also Moran et al. 2019; Gould et al. 2015; Herrero et al. 2008; Disney and Aoki 2008; Lu et al. 2020). Such M1mediated effects are thought to orchestrate multiple neuronal populations for integrating the cognitive, sensory, and motor components of a cue-evoked behavioral response. A PAMmediated amplification of such M1-dependent neuronal activity patterns would be expected to enhance and, in DL rats, rescue important aspects of the postsynaptic impact of cholinergic signals. As a result, such a treatment would be expected to benefit the ability of DL rats to more effectively employ task cues as well as utilize proprioceptive information to optimize dynamic surface traversal, and to respond to movement errors by rapidly adapting gait and balance to continue forward traversal. We tested this hypothesis using the M1 PAM TAK-071 because of this compound's relatively high selectivity for neuronal M1 receptors (Kurimoto et al. 2019; Sako et al. 2018) and based on our prior finding that this drug improves the attentional performance of rats with $\mathrm{BF}$ cholinergic lesions (Kucinski et al. 2020). In addition to testing fall propensity in rats traversing the rotating rod of the MCMCT, we also assessed their ability to traverse a rotating zigzag rod which even more severely taxes the attentional control of balance and complex movements (Kucinski et al. 2019; Kucinski et al. 2018). The main results indicate a TAK-071induced reduction in fall rate primarily in the latter condition, and specifically in interaction with "steep" zigzag entry angles that require rapid and continuous postural re-balancing and gait adjustment.

\section{Materials and methods}

Subjects Forty adult female Sprague Dawley rats (Envigo) between 2 and 3 months of age, weighing $273.79 \pm 2.78 \mathrm{~g}$ (M, SEM) at the beginning of experiments, were used for these experiments. We previously observed that male rats exhibited more falls and traversed rods more slowly than female rats, particularly on the zigzag rod (Fig. 1). In addition to evidence suggesting relatively superior attentional control of complex movement in female rats, we also found that falls in male rats were associated with their relatively higher body weights (Kucinski et al. 2019; Koshy Cherian et al. 2019; Kucinski et al. 2017; Kucinski et al. 2018). Furthermore, we previously documented beneficial attentional effects of TAK071 in male and female rats with basal forebrain cholinergic lesions, and also in female, but not male, control rats (Kucinski et al. 2020). As the present experiments were designed to determine whether these effects generalize to complex movement control, as assessed specifically by traversal of the zigzag rod, only female rats were used to optimize the documentation of such effects. As was previously discussed, such sex effects in rats may primarily inform about the cognitive-motor constraints of the efficacy of this behavioral test and the pharmacological intervention, rather than pointing to potentially equivalent sex effects in other species.

Rats were handled daily for at least 3 days prior to beginning behavioral training and received 10-20 banana pellets ( $45 \mathrm{mg}$ per pellet; BioServ) daily for 3 days prior to the start of testing. Animals were individually housed in opaque single standard cages $(27.70 \mathrm{~cm} \times 20.30 \mathrm{~cm})$ in a temperature- and humidity-controlled environment $\left(23{ }^{\circ} \mathrm{C}, 45 \%\right)$ and maintained under a 12:12-h light/dark schedule (lights on at 8:00 AM). Food (Envigo Teklad rodent diet) and water were available ad libitum. Estrous cycle was not determined. All procedures were conducted in adherence with protocols approved by the University Committee on Use and Care of Animals at the University of Michigan and in laboratories accredited by the Association for Assessment and Accreditation of Laboratory Animal Care.

\section{Michigan Complex Movement Control Task}

The MCMCT beam traversal apparatus (Kucinski et al. 2013; Kucinski et al. 2019; Kucinski et al. 2018; Sarter et al. 2014; 
Fig. 1 Zigzag rod design, nomenclature of testing conditions, and depiction of two testing conditions associated with major effects of TAK-071. The dimensions of the two zigzag segments, separated by straight rod segments (a). Rats were initially trained to traverse the (nonrotating) rod while in the flat $\left(0^{\circ} ; \mathbf{b}\right)$ and then the vertical conditions $\left(90^{\circ} ; \mathbf{c}, \mathbf{d}\right)$, followed by traversal of the rod rotating, at 5 $\mathrm{RPM}$ (or $30^{\circ} / \mathrm{s}$ ), in the clockwise (cw) or counterclockwise (cc) direction. During drug testing, rotation direction was alternated between cc and cw from run to run. As observed previously (Kucinski et al. 2019; Kucinski et al. 2018), entering a zigzag segment when at steep condition $\left(61-90^{\circ} ; \mathbf{c}-\mathbf{f}\right)$ and rotating toward a steep or vertical state $(\mathbf{e}, \mathbf{f})$ produced more falls than rotating from a steep toward a flat state $(\mathbf{g}, \mathbf{h}$; note that in $\mathbf{e}$ and $\mathbf{g}$, the rod is rotating in the cw direction, as also indicated by the arrows in $\mathbf{f}$ and $\mathbf{h})$. As detailed in "Results," TAK-071 produced robust effects in association with the angled parts being in at a "steep" angle upon entrance as exemplified in $\mathbf{e}$

a

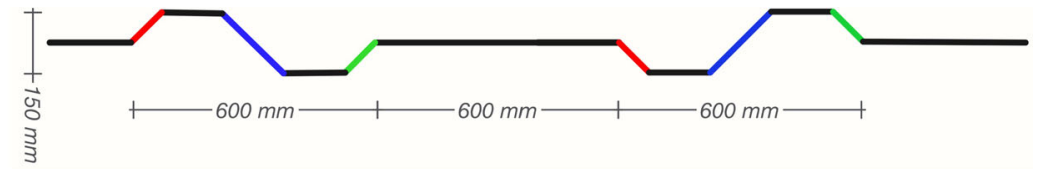

b

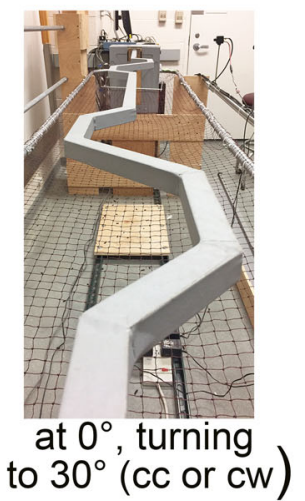

C

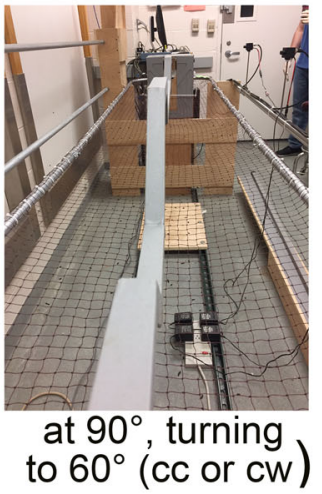

d

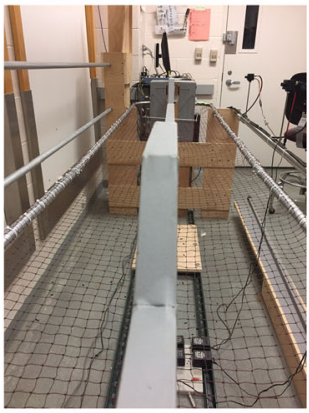

at $90^{\circ}$, turning to $60^{\circ}$ (cc or $\mathrm{cW}$ ) e

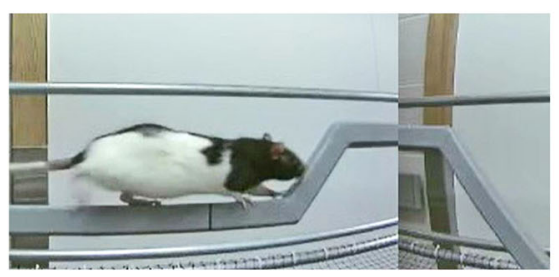

g

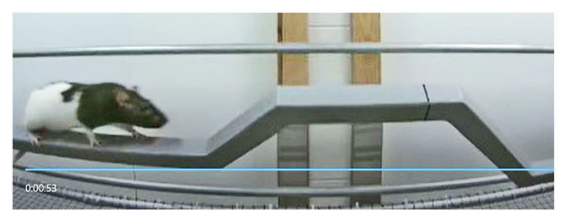

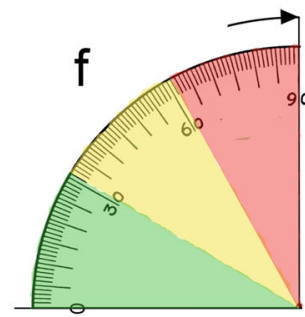

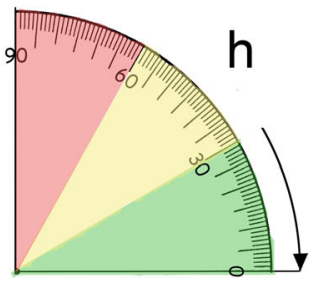

Koshy Cherian et al. 2019; Avila et al. 2020) assesses the ability of rats to perform attention-demanding beam traversals and correct for stepping errors while crossing a "straight" narrow rod or a bended rod surface ("zigzag"; see below). The rods were $3.0 \mathrm{~m}$ in length (square; side lengths $1.59 \mathrm{~cm}$ ), made from aluminum tubing covered with gray gaffer's tape for traction. There was also a plank surface $(13.3 \mathrm{~cm}$ width) that could be placed directly on top of the straight rod and fitted firmly in place inside edges of the support towers used to familiarize rats with traversing from goal box to goal box. The straight and zigzag rods could be rotated using a 12 VDC electric motor controlled remotely by a pulse width modulator which was able to adjust the speed of rotation (up to 10 RPM) and switch the direction of rotation. A safety net was suspended $20 \mathrm{~cm}$ under the beam to catch the rats during falls. Two identical end stations were situated on top the support towers at opposite ends of the beam. These stations consisted of a $30 \times 25-\mathrm{cm}$ platform with a $3-\mathrm{cm}$ diameter copper cup embedded in the floors. During training and testing, rats were given a banana pellet placed inside the cups in the goal boxes following each traversal. Each goal box was surrounded by a retractable wall structure $(23 \mathrm{~cm}$ height in raised position) to allow for conversion from an open platform to a boxed structure. On the wall facing the beam of each box structure, a 9-cm wide opening allowed the rats access to and from the beam. The walls were raised and lowered mechanically with a 12 VDC electric motor actuated remotely by a toggle switch. Lowering the walls and thereby turning the box into an open-field platform was sufficient for rats to initiate beam traversal to enter the opposite box (with walls up) and consume the banana pellet. Between traversals, the walls of the goal boxed remained in the up-position for $45 \mathrm{~s}$. Videos of traversals were recorded with 4 bullet Marshall 1080-HD-DI model CV500 Series cameras (B-30/25P frame rate/59.94i) mounted on the net frame parallel to one side of the beam. The videos were converted to a single feed using a quad SDI to HDMI multiviewer (Matrox MicroQuad) and viewed directly on a PC using Elgato Game Capture HD software. 
Straight rotating rod Performance measures were falls and traversal time on the straight rotating rod. These measures were analyzed in the same manner as previously described (Kucinski et al. 2017; Kucinski et al. 2013). Rats were allowed to fall into the net and placed back onto the rod or, upon an imminent fall, the experimenter assisted the rat in regaining balance on the rod, and no further falls were counted until the rat regained a balanced posture and resumed forward movement. In addition to complete falls from the straight rod ("major falls"), we also counted as falls when the rat would completely cease forward movement and assume a slouched, nearly immobile position on the rod ("minor falls"). In either case, traversal time was corrected for fall-related disruptions of forward movement. In the overwhelming number of cases, rats were able to regain forward movement within $2 \mathrm{~s}$ of being placed back onto the rod. Because multiple falls could occur during a run, falls per run were used as a metric for fall rates. We previously reported high inter-rater reliability for extracting the behavioral measures from videotaped MCMCT runs (Avila et al. 2020).

Zigzag rotating rod A zigzag rod variation was designed to particularly severely tax complex movement control (Kucinski et al. 2019; Kucinski et al. 2018). This rod (Fig. 1) included two zigzag sections that were each $0.6 \mathrm{~m}$ in length and placed $0.6 \mathrm{~m}$ from either end of the rod and separated by a 0.6-m-long straight rod in the middle (Fig. 1a). Each zigzag section included three angled sections of varying lengths (two $10.7 \mathrm{~cm}$ and one $21.5 \mathrm{~cm}$ in length) that bended at $45^{\circ}$ angles from the horizontal plane of the rod and that connected to two straight sections (each $15 \mathrm{~cm}$ in length) that rested $3 \mathrm{~cm}$ above or below the plane, the vertical plane of the rod. The diameter of the rod tube remained $2.54 \mathrm{~cm}$ across the entire rod.

The rod could be held stationary either horizontally, so the zigzag sections protruded to the sides thus forcing rats to turn left or right while traversing (Fig. 1b), or so the zigzag sections faced up and down vertically (Fig. 1c, d), or rotated (5 RPM) so that rats were required to perform sideways and vertical movements while navigating over the zigzag sections. In contrast to falls from the straight rod, "minor falls" from the zigzag portions did not occur. Furthermore, performance on the straight parts of the zigzag rod was not analyzed. Thus, fall rates from the two rod types are not directly comparable. In addition to falls, we determined, for each entry, onto the first and second angled part of the zigzag sections: (1) the angle of elevation above or below the horizontal plane at the time when rats entered (an entry was scored when the rat placed its forepaws onto such a section and continued to move onto the angled part), and (2) whether the angled part was rotating upwards (Fig. 1e, f) or downwards (Fig. 1g, h). Angles of elevation at the time of entrance (forepaws placed onto an angled section) were categorized as flat $\left(0-30^{\circ}\right.$ away from the horizontal or flat level shown in Fig. 1b); medium steep (31-60 $)$; or steep (61$90^{\circ}$, with $90^{\circ}$ depicting the zigzag section in vertical position, facing upward or downward as shown in Fig. 1c, d). Rotation direction (upwards or downwards) was further defined depending on whether the angled part rotated toward a steeper state (Fig. 1e, f) or a flatter state (Fig. 1g, h).

\section{Training and testing regimen}

Prior to receiving DL or sham surgeries (below), rats were habituated to the traversal apparatus over a 5-day test sequence, including runs on the plank, nonrotating straight rod, rotating straight rod (5 RPM), and the zigzag surface (nonrotating runs in the vertical and horizontal positions and rotating runs at 5 RPM; Table 1).

Following completion of the pre-surgery MCMCT practice, animals were randomly distributed into two groups, designated to either receive DL surgery $(n=30)$ or sham surgery $(n=10)$. Recovery from surgery was between 5 and 10 weeks; this relatively long and variable period of time between surgeries and post-surgery testing was due to the COVID-19-associated cessation of laboratory research. Fourteen rats were tested following a 5week break and 26 rats were tested following a 10 -week period. A post hoc analysis of a potential effect of break duration on fall rates on both the rotating straight rod (combined 3 days) and the zigzag rod (combined two days) did not reveal a main effect of interactions with group (all $F<0.84, P>0.37$ ). Among the rats which were tested following a 10 -week break, the data from 3

Table 1 Sequence of MCMCT testing conditions

\begin{tabular}{|c|c|c|c|}
\hline Day & Beam & Rotating (speed) & Number of runs \\
\hline \multicolumn{4}{|c|}{ Pre-surgery } \\
\hline 1 & Plank & $\mathrm{n} / \mathrm{a}$ & 6 \\
\hline 2 & Straight rod & 0 RPM & 6 \\
\hline 3 & $\mathrm{cc}$ rotating rod & 5 RPM & 6 \\
\hline \multirow[t]{2}{*}{4} & Zigzag flat & $\mathrm{n} / \mathrm{a}$ & 3 \\
\hline & Zigzag vertical & & 3 \\
\hline 5 & Zigzag rotating — alt direction & 5 & 6 \\
\hline \multicolumn{4}{|c|}{ Post-surgery and TAK-071 testing } \\
\hline \multirow[t]{2}{*}{1} & Plank & $\mathrm{n} / \mathrm{a}$ & 3 \\
\hline & Straight rod & 0 RPM & 3 \\
\hline 2 & cc-rotating rod & 8 RPM & 6 \\
\hline 3 & cw-rotating rod & 8 RPM & 6 \\
\hline 4 & Alt direction rotating rod & 8 RPM & 6 \\
\hline \multirow[t]{2}{*}{5} & Zigzag flat & $\mathrm{n} / \mathrm{a}$ & 3 \\
\hline & Zigzag vertical & & 3 \\
\hline 6 & Zigzag rotating - alt direction & 5 RPM & 6 \\
\hline 7 & Zigzag rotating—alt direction & 5 RPM & 6 \\
\hline
\end{tabular}


sham-operated, and 4 DL rats (one treated with vehicle, two with $0.1 \mathrm{mg} / \mathrm{kg}$, and one with $0.3 \mathrm{mg} / \mathrm{kg}$ TAK-071), were removed from the final analysis because these rats failed to traverse the rods. In addition, one sham-operated and one DL/vehicle rat did not traverse the zigzag rod on the final day (day 7) of testing.

Following surgery, the $30 \mathrm{DL}$ rats were randomly divided into 3 groups (10 each) to be treated with either vehicle or 0.1 $\mathrm{mg} / \mathrm{kg}$ or $0.3 \mathrm{mg} / \mathrm{kg}$ TAK- 071 . Sham-operated rats were treated with vehicle (see below). All rats then underwent a 7-day traversal test sequence (Table 1), including runs on the plank and straight rod surfaces (for re-acclimatization to the testing conditions, day 1 ), rotating straight rods (days 2,3 , and 4 ), and the zigzag rods (days 5, 6, and 7).

\section{TAK-071}

TAK-071 (Takeda Pharmaceutical Company; Lot \# M071004) was dissolved in $0.5 \%$ methyl cellulose in DI water (vehicle). Vehicle and a $0.1-\mathrm{mg} / \mathrm{mL}$ stock solution were prepared on the first day of drug administration and kept cool and out of light during the 7-day test sequence. The stability of this preparation was determined by Takeda Pharmaceutical Company (Yuta Arai, Takeda Pharmaceutical Company Limited, unpublished data). Animals were familiarized with gavage administration procedures for five consecutive days prior to the onset of the drug testing period. Rats were administered vehicle $(3.0 \mathrm{~mL} / \mathrm{kg})$ or drug $(0.1$ or $0.3 \mathrm{mg} / \mathrm{kg})$ via oral gavage $30 \mathrm{~min}$ prior to the onset of the daily test session, for a total of 7 days. The two doses for testing were selected based on pharmacological and pharmacokinetic data (Kurimoto et al. 2019; Sako et al. 2018). Specifically, following oral administration of $0.3 \mathrm{mg} / \mathrm{kg}$, peak brain concentration $\left(C_{\max }\right)$ was $0.11 \mu \mathrm{M}$ (for plasma and brain concentrations following oral administration of $0.1-10 \mathrm{mg} / \mathrm{kg}$ of TAK-071 in rats, see Table S2 in Sako et al. 2018). The brain concentration following oral administration was found to be well above the half maximal concentration for PAM at M1 AChR (2.7 $\mathrm{nM}$ ), but below concentrations required for PAM at M2-M5 AChRs (> 1000 nM; Sako et al. 2018). TAK-071 is a highly selective PAM, relative to agonist effects at the M1 AChR (EC50: 520 nM; Sako et al. 2018). Following oral administration of 0.1 and $0.3 \mathrm{mg} / \mathrm{kg}$, the half-life of TAK-071 ( $\left.T_{\max }: 1.7 \mathrm{~h}\right)$ extended well beyond the period of behavioral testing in the current experiment (3045 min following administration). Evidence describing the effects of semi-chronic administration of TAK-071 on plasma and brain concentrations appears unavailable. Finally, for the present experiment, the two doses were selected based on our prior study indicating efficacy of these doses, administered over 6 days, in rats with cholinergic lesions and performing a sustained attention task (Kucinski et al. 2020).

\section{Surgeries}

Rats were anesthetized with isoflurane gas (induction, 4-5\%; maintenance, 2-3\%) delivered at $0.6 \mathrm{~L} / \mathrm{min} \mathrm{O}_{2}$ using a SurgiVet Isotec 4 Anesthesia Vaporizer and mounted to a stereotaxic instrument (David Kopf Instruments, Tujunga, CA). Animal temperatures were maintained at $37{ }^{\circ} \mathrm{C}$ using Deltaphase isothermal pads (Braintree Scientific). Ophthalmic ointment was provided for eye lubrication. A subcutaneous injection of $1 \mathrm{~mL} / 100 \mathrm{~g} 0.9 \% \mathrm{NaCl}$ solution was administered to prevent hypovolemia and hemodynamic instability. Rats also received carprofen $(5 \mathrm{mg} / \mathrm{kg}$; subcutaneous; Henry Schein) for pain relief just prior to individual surgeries. Rats received bilateral bolus infusions $(10 \mu \mathrm{g} / \mu \mathrm{L} ; 1$ $\mu \mathrm{L} /$ hemisphere) of the neurotoxin 6-hydoxydopamine (6OHDA; Sigma-Aldrich, MO, $n=30)$ or vehicle $(1 \mathrm{mg} / \mathrm{mL}$ ascorbic acid in $0.9 \% \mathrm{NaCl} ; n=10$ ) into two sites of the striatum (bregma A/P: +1.2 or $+0.2 \mathrm{~mm}, \mathrm{M} / \mathrm{L}: \pm 2.5$ or \pm $3.0 \mathrm{~mm}, \mathrm{D} / \mathrm{V}:-5.0 \mathrm{~mm}$ from dura). Rats were also injected with desipramine hydrochloride $(10 \mathrm{mg} / \mathrm{kg}$; i.p.; SigmaAldrich) $30 \mathrm{~min}$ prior to neurotoxin infusions to protect noradrenergic neurons (Breese and Traylor 1971). Rats received bilateral infusions of the cholino-specific immunotoxin 192 IgG-saporin $(200 \mathrm{ng} / \mu \mathrm{L} ; 0.8 \mu \mathrm{L} /$ hemisphere; Advanced Targeting Systems, CA, $n=30$ ) or an equal volume of artificial cerebral spinal fluid $(n=10)$ into the nucleus basalis and substantia innominata of the basal forebrain (BF; bregma A/P: $-0.8 \mathrm{~mm}, \mathrm{M} / \mathrm{L}: \pm 2.9 \mathrm{~mm}, \mathrm{D} / \mathrm{V}:-7.8 \mathrm{~mm}$ ). For all infusions, an additional $8 \mathrm{~min}$ was allowed for diffusion before the needle was removed. Nonabsorbable nylon sutures were used to close the incisions. Topical antibiotic (Neosporin) was applied to the wounds. Rats received one subsequent dose of carprofen $(5 \mathrm{mg} / \mathrm{kg}) 1$ day postsurgery, followed by 5-10 weeks of recovery period before the initiation of behavioral testing.

\section{Histology}

Following the completion of the behavioral testing, rats were deeply anesthetized with a lethal dose of sodium pentobarbital ( $270 \mathrm{mg} / \mathrm{kg}$, i.p.) and transcardially perfused with phosphatebuffered solution (PBS) followed by $4 \%$ paraformaldehyde in $0.15 \mathrm{M}$ sodium-phosphate solution, $\mathrm{pH}$ 7.4. Brains were extracted and postfixed in $4 \%$ paraformaldehyde for $24 \mathrm{~h}$, rinsed with PBS, and then placed in $30 \%$ sucrose solution until they sank. Brains were sectioned into $35-\mu \mathrm{m}$-thick slices using a freezing microtome (CM 2000R; Leica) and stored in cryoprotectant until further histological processing. Sections from lesioned and sham-lesioned rats were stained, separately, for the visualization of acetylcholinesterase ( $\mathrm{AChE}$ )-positive fibers in the cortex and the presence of striatal tyrosine hydroxylase (TH). The density of AChE-positive fibers in cortex and TH depletions were quantified as described previously (Avila 
et al. 2020; Koshy Cherian et al. 2019; Kucinski et al. 2013). Briefly, a $4 \times 4$ grid of $30-\mu \mathrm{m}$ (side length) squares was digitally superimposed photographs of AChE-positive fibers in layers $5 / 6$ of primary motor cortex ( $\times 40$ magnification). Labeled fibers which crossed one of the lines of the superimposed grid were counted using the ImageJ multipoint tool. The extent of cholinergic loss was quantified for each DL rats by subtracting their individual fiber count from the mean fiber count from sham-lesioned rats (for both hemispheres and then averaged to a single value per rat). The degree of AChEpositive fiber loss was scored from 5 to 1 , with 5 indicating the greatest loss of AChE-positive fibers (5: > 90\% loss; 4: > $80 \% ; 3:>70 \% ; 2:>60 \% ; 1: 30-50 \%$ loss). Photographs of $\mathrm{TH}$ immuno-stained sections were taken at $1.25 \times$ from sections at approximately $500 \mu \mathrm{m}$ posterior and anterior from the infusion sites at $0.2 \mathrm{AP}$ to 1.2 AP. The extent and location of the bleaching of TH stains was rated. TH-IR losses greater than $2.00 \mathrm{~mm}$ (dorsal-ventral plane) $\times 0.5 \mathrm{~mm}$ (mediallateral plane) $\times 0.5 \mathrm{~mm}$ (anterior-posterior plane) were assigned the highest score (5). Lower scores $(\leq 3)$ were assigned to smaller lesion sizes. We previously determined that TH losses in the mediodorsal part of the striatum, overlapping with the target field of prefrontal cortical projections, are necessary and sufficient to produce falls in DL rats (Kucinski et al. 2015; Kucinski et al. 2013). TH losses centered closest and mostly restricted to the mediodorsal caudate nucleus were assigned the highest score (5), whereas lesions spreading more laterally received scores $\leq 3$. The size and placement scores for both hemispheres were averaged to a single DA lesion score for each DL rats. Finally, a composite lesion score was calculated for each DL rats by multiplying the $\mathrm{AChE}$ and $\mathrm{TH}$ loss depletion score. Thus, a near-complete loss of cortical AChEpositive fibers combined with large mediodorsal striatal TH depletions would receive a rating of 25 .

Statistical analyses For runs on the straight rotating rod, falls and traversal time were compared between the groups (shamvehicle, DL-vehicle, DL-0.1 mg/kg TAK-071, and DL-0.3 $\mathrm{mg} / \mathrm{kg}$ TAK-071) using the data from the 4 th day of treatment and from runs across which the rotation direction of the rod was alternated (ANOVA). Falls during zigzag beam traversals on days 6 and 7 likewise were analyzed using ANOVA, with alpha set at $0.05 / 2$ for conducting separate analyses. For the post hoc investigation of the impact of entry angle category and rotation direction, falls obtained from the last day of testing were computed over the number of entries per category and over all 6 runs, and analyzed using repeated measures ANOVA of the effects of angle category and group. Post hoc multiple comparisons were conducted using Fisher's least significance difference (LSD) test. Statistical analyses were performed using the SPSS for Windows (version 17.0: SPSS). Assumptions underlying the statistical model were assessed. In cases of violation of the sphericity assumption, Huyhn-Feldt-corrected $F$ values, along with uncorrected degrees of freedom, are given. Alpha was set at 0.05 except for the analysis of falls from days 6 and 7 (above). Exact $P$ values are reported as recommended previously (Greenwald et al. 1996; Sarter and Fritschy 2008). Effect sizes (Cohen's $d$ ) were calculated for major significant effects and classified in accordance to Cohen (1988).

\section{Results}

\section{Straight rotating rod performance in DL rats and effects of TAK-071}

Following recovery from surgeries, animals underwent drug testing with TAK-071 or vehicle over a 7 (consecutive)-day traversal sequence (Table 1). TAK-071 or vehicle was administered via gavage $30 \mathrm{~min}$ prior to the onset of MCMCT testing on each of the 7 test days. On the first day of testing, rats were refamiliarized with traversing the plank and nonrotating straight rod surfaces. On the second, third, and fourth days, straight rotating rod traversals were assessed (6 runs per day; clockwise (cc) day 1, counterclockwise (cw) day 2, alt direction day 3; 8 RPM for all days; see Fig. 2a for an illustration of the straight rod testing apparatus). Falls and traversal time were extracted from these runs and compared between the four groups (shams treated with vehicle, S-V; DL rats treated with vehicle, DL-V; DL rats treated with $0.1 \mathrm{mg} / \mathrm{kg}$, DL-0.1; or with $0.3 \mathrm{mg} / \mathrm{kg}$ TAK-071, DL-0.3), with rotating direction as a within-subjects factor. As cc- and cw-rotating rod traversal was tested early into the treatment regimen, on days 2 and 3, the analysis of straight rotating rod performance was limited to the test of the relatively more demanding condition on the 4th day of treatment, that is, when alternating the rod direction from run to run.

As expected, based on our prior experiments on the performance of DL rats, DL-V rats fell more frequently than S-V rats (main effect of group: $F(3,32)=3.13, P=0.04$; post hoc comparison (LSD): $P=0.03$; Cohen's $d$ : 0.93). Furthermore, DL-0.1 rats fell nearly significantly less than DL-V rats $(P=0.05$; Fig. $1 \mathrm{~b})$. The size of the near-significant effect of the lower dose of TAK-071 $(d: 0.89)$ was "large" as classified by Cohen (1988).

DL-V rats appeared to require more time traversing the alternatingly rotating rod than S-V and DL-0.1 rats, but the effect of group did not reach significance $(F(3,32)=1.81, P=0.17$; Fig. $1 \mathrm{c})$.

Falls and traversal time were significantly correlated across all groups of rats $\left(R^{2}-0.74, P<0.001\right)$ and, with the exception of DL-0.3 rats, also within individual groups (S-V, DL-V, DL-0.1 groups: $R^{2}>0.65, P<0.02$; DL-0.3: $R^{2}=0.35, P=$ 0.09; Fig. 2d). The lack of a significant correlation between 


\section{a}

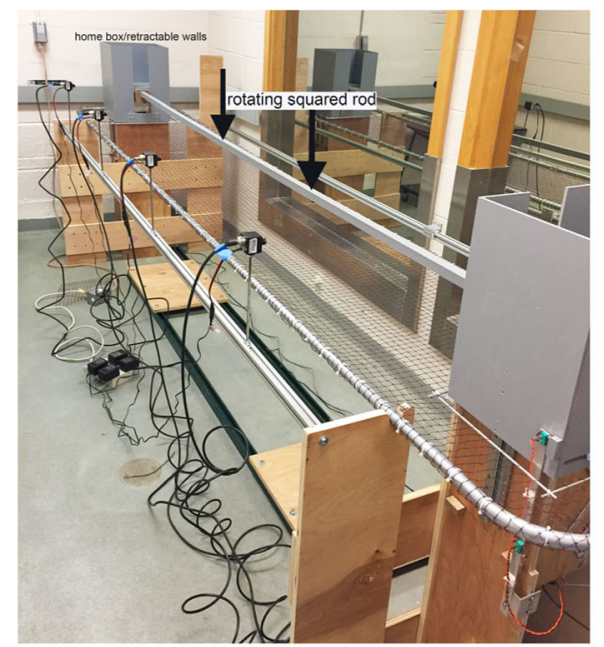

C

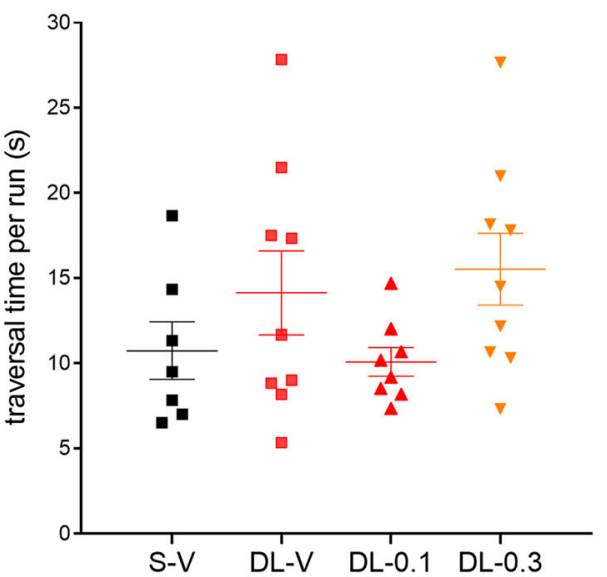

Fig. 2 DL-0.1 rats fell less frequently than DL-V rats while traversing the alternatingly rotating rod. a The MCMCT with the straight rod installed (see black arrows). Note also the home boxes on either end of the rod, the walls of which could be lowered to produce an open field, thereby motivating the rat to traverse across the rod to the opposite box (walls raised and baited with a banana pellet). b ANOVA of fall rates of the 4 groups indicated a main effect (see "Results" for ANOVAs) and post hoc multiple comparisons $(* P<0.05)$ reflected that DL-V rats fell more

traversal time and falls in DL-0.3 rats suggested that, in contrast to the effects of the lower dose of TAK-071, DL-0.3 rats continued to traverse the rod as slowly as DL-V rats while falling at a (nonsignificantly) lower rate than DL-V rats.

\section{Zigzag rod performance in DL rats and effects of TAK- 071}

On the 5th day of testing (Table 1), rats were refamiliarized with traversing the zigzag rod fixed in the horizontal and vertical position. Next, falls and traversal time were assessed across two consecutive days based on traversals of the alternatingly rotating zigzag rod (5 RPM). Separate analyses for falls from days 6 and 7 , with alpha set at $0.05 / 2$, indicated a main effect of group on falls during the second day of rotating
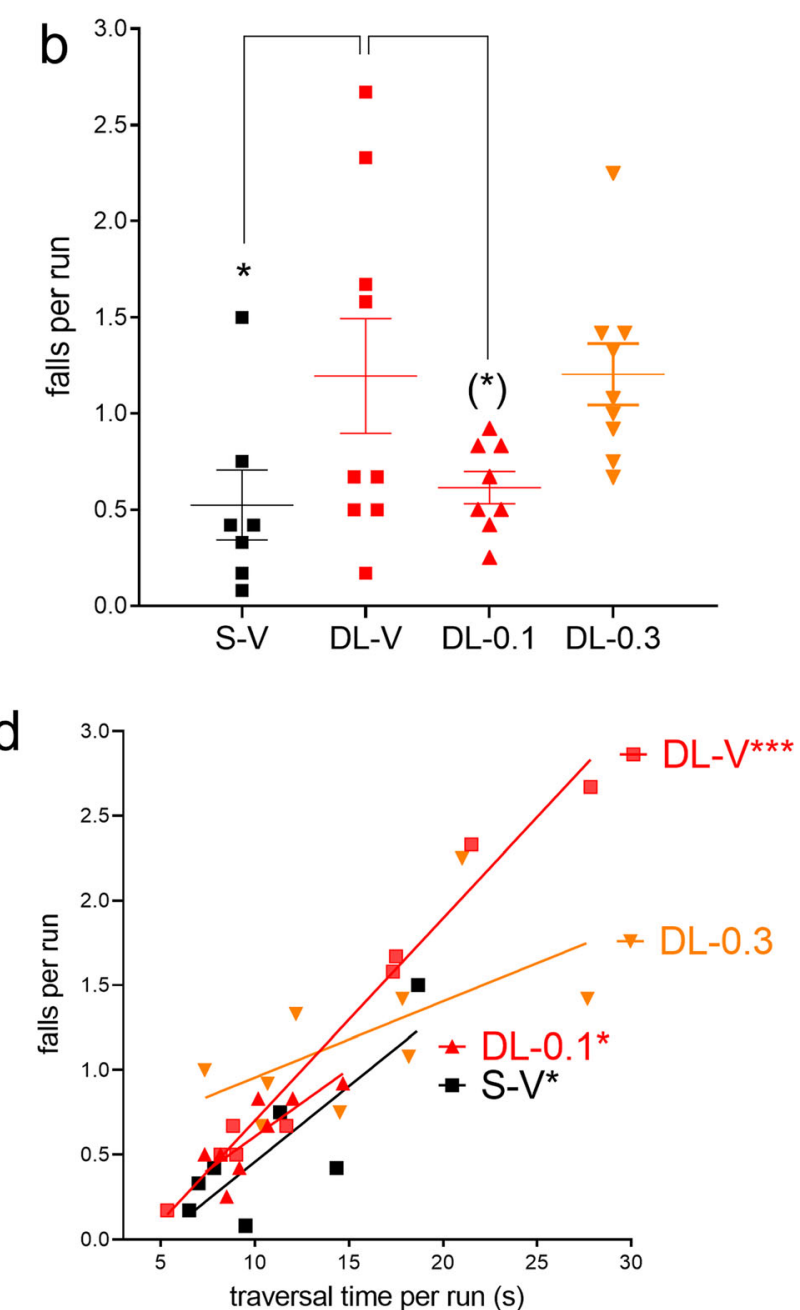

frequently than S-V and DL-0.1 rats (for the latter comparison, (*) depicts $P=0.05$ but the effect size $(d=0.89)$ was "large" (Cohen 1988). c The pattern of traversal times across the four groups mirrored the distribution of fall rates but effects of group did not reach significance. $\mathbf{d}$ Fall rates and traversal time were significantly correlated in all groups but the DL- 0.3 rats, suggesting that these rats did not reduce rod traversal speed while exhibiting a (nonsignificant) decrease in fall rates

zigzag rod testing $(F(3,30)=5.51, P=0.004)$ but not on the first test of this condition $(F(3,32)=0.96, P=0.43)$. Multiple comparisons indicated that on the second day of traversing the rotating zigzag rod, DL-V rats fell more frequently than all other groups (all $P<0.03$; all Cohen's $d>1.03$; Fig. 3a).

To further explore the specific zigzag conditions which were associated with high falls in DL-V rats and the reduction in falls in DL rats treated with TAK-071, we applied a previously established sorting scheme of the status of the zigzag segments (see Fig. 1 for an abbreviated illustration of major zigzag conditions; for more details, see Kucinski et al. 2018). This analysis was based on a total of 744 entries to zigzag segments, obtained from $6 \mathrm{~S}-\mathrm{V}, 8 \mathrm{DL}-\mathrm{V}, 8 \mathrm{DL}-0.1$, and 9 DL-0.3 rats (24 entries per rat), and from the second day of alternatingly rotating zigzag runs. 


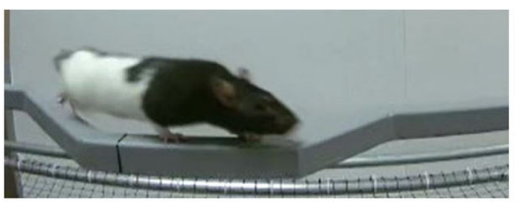

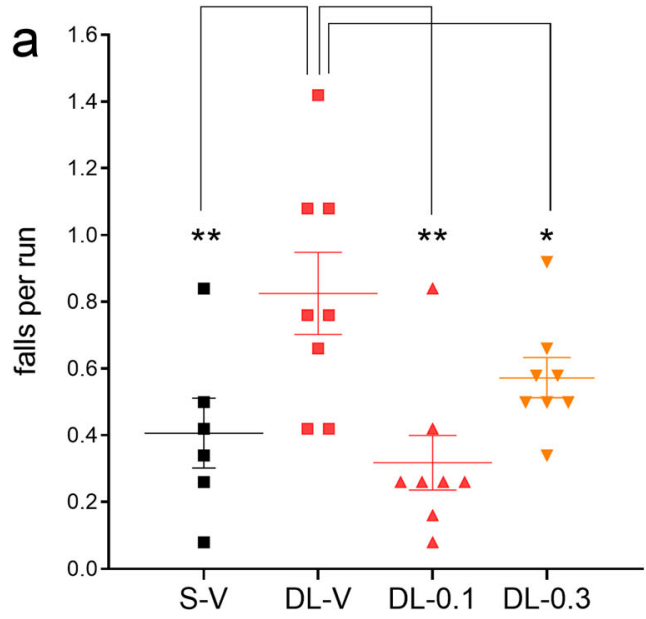

Fig. 3 a Falls during traversals of the rotating zigzag rod (day 7). DL-V rats fell significantly more often than all other three groups of rats (note that fall rates from the straight and zigzag rods are not directly comparable; see "Methods"). Further investigation of the state of the zigzag segment which fostered falls indicated that they were mainly evoked when the angled part of the zigzag was at a "steep" condition $\left(61-90^{\circ}\right.$; see red segments in the right insert in $\mathbf{b}$; note that in $\mathbf{a}$, falls are expressed by run and in b per entry category and over all 6 runs). The impairment of DL-V

Our initial analysis focused on the possibility of a robust differentiation between the groups for entries into the zigzag portion when the bent parts were sloped $61-90^{\circ}$ above or below the horizontal (or "steep"; Fig. 1c, d), because this condition was previously identified as associated with more falls than less steep entry states (Kucinski et al. 2019; Kucinski et al. 2018). First, the total number of entries into the bent sections at one of three angle categories (flat: $0-30^{\circ}$, medium: $31-60^{\circ}$, steep: $\left.61-90^{\circ}\right)$ did not differ between the groups (group $\times$ angle category; $\left(\chi^{2}(6)=8.63, P=0.20\right.$ ). This finding rejects the possibility that certain entry angle categories were avoided by individual groups of rats.

Next, we computed the number of falls per entry category (Fig. 3b). ANOVA of the effects of group and entry angle category ("flat" or $0-30^{\circ}$, see green segments on the left of Fig. 3b; "medium steep" or $31-60^{\circ}$, see yellow segments in the middle of Fig. 3; "steep" or $61-90^{\circ}$, see red segments on the right of Fig. 3b) indicated a significant interaction between the effects of the two factors $(F(6,54)=2.84, P=0.02$; Fig. $3 b)$. In addition, this analysis, of falls per entry category and summed up of all 6 runs (and thus different to the falls per run shown in Fig. 3a), indicated a trend for a main effect of group $(F(3,27)=2.89, P=0.056)$, mirroring the data pattern shown in Fig. 3a, and a main effect of entry category $(F(2,54)=$ $28.76, P<0.001$; Fig. 3b). To identify the locus of the interaction between the effects of group and angle category, one-

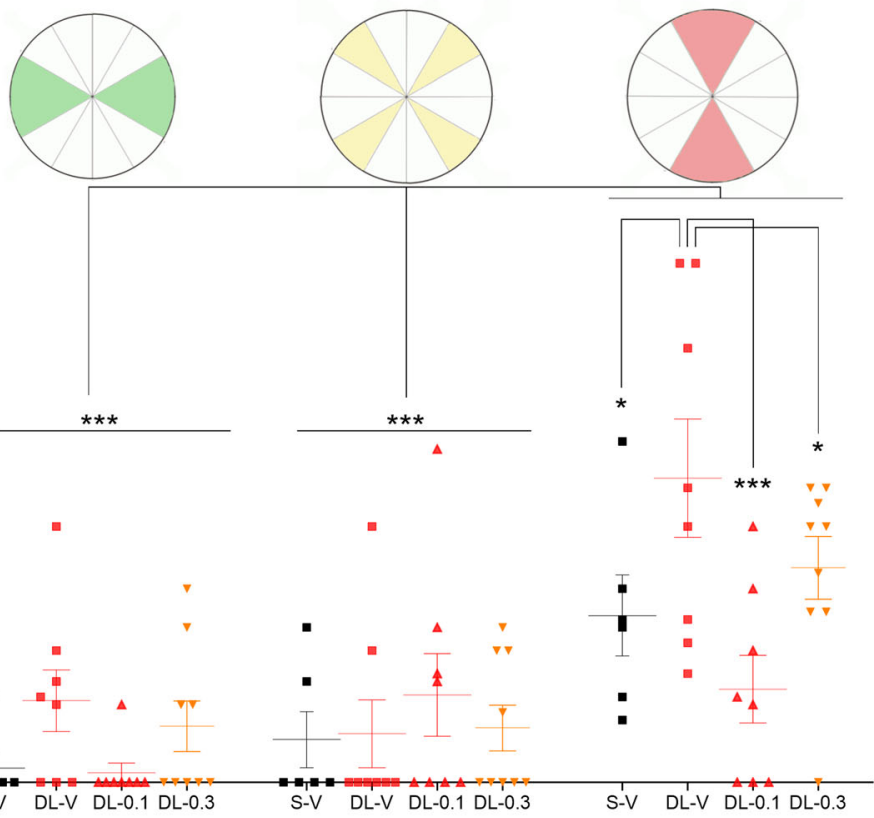

rats thus manifested selectively in association with steep entry angles, and the reduction of falls in DL rats treated with TAK-071 likewise was restricted to this entry angle category. Whether the steep-angled segment of the zigzag rotated toward an even steeper versus a flatter angle did not significantly influence the fall rates occurring in association with steep entry angles (see "Results" for ANOVA; post hoc comparisons: $* P<$ $0.05 ; * * P<0.01 ; * * * P<0.001)$

way ANOVAs on the effects of group per angle category indicated a significant effect for the steep $(F(3,30)=4.48, P$ $=0.01$ ) but not the two less steep angle categories (both $P>$ 0.11 ). Thus, these analyses indicated that all groups of rats fell relatively more frequently when entering an angled part in the steep orientation, and that this condition revealed the relatively higher fall rate of DL rats as well as a reduction of falls in DL rats treated with TAK-071 (Fig. 3b; Cohen's $d$ for DL-V versus $0.1 \mathrm{mg} / \mathrm{kg}$ : $1.56 ; 0.3 \mathrm{mg} / \mathrm{kg}$ : 0.67). Finally, exploration of the impact of rotation direction (turning the angled part into an even steeper versus a flatter condition) did not provide further insights into the propensity for falls associated with the steep entry angle category (main effect and interaction, both $P>0.27$ ).

\section{Histological findings}

The density of AChE-positive fibers in cortex was significantly reduced in all DL groups, with no significant differences between the three groups of DL rats $(F(3,31)=48.95, P<$ 0.001 (S-V: $65.64 \pm 4.35$ fibers; DL-V: $21.83 \pm 1.20$; DL-0.1: $27.64 \pm 2.52$; DL-0.3: $24.56 \pm 2.76$; multiple comparisons indicated that fiber counts in all DL rats were significantly lower than in $\mathrm{S}-\mathrm{V}$ rats, all three $P<0.001$ ). On average, AChE-positive fiber losses received a rating of $2.92 \pm 0.22$ (see "Materials and methods"), reflecting a loss of about $60 \%$ 
of cholinergic inputs. Likewise, neither the size nor the location of mediodorsal striatal TH losses differed between the three DL groups (size: $F(2,24)=0.08, P=0.93$; score: 2.09 \pm 0.15 ; location: $F(2,24)=0.97, P=0.40$ (score: $3.92 \pm 0.17$ ). TH losses in DL rats were previously found to be correlated with fall rates (Avila et al. 2020; Koshy Cherian et al. 2019; Kucinski et al. 2017; Kucinski et al. 2013). The present data did not indicate a significant correlation between TH losses and falls in DL-V rats, likely because of a relatively limited variability of TH losses in these rats. Losses of cortical AChEpositive fibers and striatal $\mathrm{TH}$-immunoractivity were extensively illustrated in prior reports describing experiments involving DL rats (see Avila et al. 2020; Koshy Cherian et al. 2019; Kucinski and Sarter 2015; Kucinski et al. 2015; Kucinski et al. 2013, 2017).

\section{Discussion}

DL rats model the combined BF cholinergic and mediodorsal striatal dopaminergic losses which characterize PD fallers. Fall rates were measured in DL rats traversing a rotating straight rod and a rotating zigzag rod. As was expected, DL rats fell more frequently than sham-operated rats in both conditions. Administration of the lower dose of TAK-071, daily over a 7-day period and prior to daily test sessions, nearly significantly reduced falls during rotating rod traversal $(P=$ 0.05; Cohen's $d: 0.89$ ). Both doses of the M1 PAM reduced falls occurring during zigzag rod traversal. In the latter test, the number of falls in DL rats was particularly high, and the M1 PAM was robustly beneficial, when the angled part of the zig zag rod was at a steep, near vertical angle at the time the rats entered a zigzag segment.

The current experiments were conducted using only female rats because prior experiments indicated a relatively greater degree of complex movement control by females, particularly when traversing the zigzag rod (Kucinski et al. 2018; Kucinski et al. 2019). Moreover, our prior study on the effects of TAK071 on sustained attention performance in rats with BF cholinergic lesions indicated a broader efficacy of the compound in females (Kucinski et al. 2020). Although there is evidence indicating that female humans likewise exert relatively superior cognitive control of complex movements (Pereira et al. 2013; Hollman et al. 2011; Lissek et al. 2007), these effects of sex in rats may primarily inform about effects of neuronal and pharmacological manipulations in interactions with biological variations of complex movement control.

The fall-reducing effect of the lower dose of TAK-071 on rotating straight rod traversal in DL rats was associated with a nonsignificant decrease in traversal speed when compared with DL-V rats (Fig. 2c). Moreover, and mirroring sham-operated, non-treated rats $(\mathrm{S}-\mathrm{V})$, a significant correlation between traversal speed and fall rate was found. In contrast, these two measures were not correlated in DL rats treated with the higher dose of TAK-071, that is, in DL-0.3 rats a trend for a decrease in falls was not associated with relatively faster traversal speed (see the "flatter" regression line for DL-0.3 rats in Fig. 2d). As is the case in older humans and PD patients (Holtzer et al. 2014; Coelho et al. 2012; Plotnik et al. 2011; Springer et al. 2006), our collective evidence suggests that in rats, slower traversal or gait speed reflects limitations in the attentional control of movement, rather than an impairment in "purely" motor functions. The conclusion that the lower dose of TAK-071 acted primarily by restoring the top-down control of movement, perhaps by benefiting the (residual) ability of DL rats to process task- and proprioceptive cues, is consistent with the efficacy of this dose on the attention of rats with solely cholinergic losses (Kucinski et al. 2020).

In contrast to fall rates on straight rotating rod, fall rates from the rotating zigzag rod benefited from both doses of TAK-071. Traversing this rod, with the direction of rotation alternating from trial to trial, arguably taxes gait and balance control robustly more than the straight rotating rod. Moreover, falls across all experimental groups differentiated specifically when the angled part was at a steep angle upon entering (Fig. 3b). At a "flatter" angle (as illustrated in Fig. 1b), rats appeared to be able to maintain a seemingly regular paw placement rhythm and a stable patterning of movement while traversing the zigzag segment. At a steep angle, and presumably triggered by mismatches between habitually pre-programmed movement sequences and the balancing and stepping demands of walking up or down at a steep and rapidly changing angle $\left(30^{\circ} / \mathrm{s}\right)$, such patterning may have been disrupted. Upon entering a steep-angled part, rats often displayed a momentarily "slouched" position, perhaps indicating such disruption of stable movement pattern. Thus, performance on the steep angle condition of the zigzag may serve as a particularly robust indicator of the ability to revise the movement plan and, compared to more flat positions of the zigzag or traversing the rotating straight rod, to integrate information about dynamic surfaces, posture, and balance with ongoing gait. In other words, the steep zigzag condition may potently trigger a robust attentional shift toward movement (re)programming (see also Wong et al. 2015).

The reduction of falls from the zigzag rod seen in DL rats treated with TAK-071 was observed on the second, but not the first, day of testing this rod when rotating in alternating direction from trial to trial (that is, on day 7 , but not day 6 , on Table 1). This finding suggests that the effects of the drug treatment on day 7 depended on practicing traversal of prior rod conditions in the presence of drug. Although potential interactions between the present treatment regimen and the escalating demands imposed by the changing task conditions (Table 1) complicate the interpretation of behavioral effects, such interactions reflect that treatments which enhance behavioral functions, or even rescue behavioral deficits, are unlikely 
to install instant, "magic bullet"-like, improvements in behavior. Rather, such treatments would be assumed to act via progressively influencing the bidirectional interplay between neuronal activity and behavior (Sarter 2006).

Although great caution is warranted when using the present, relatively limited data set from rats to predict clinical efficacy of the compound, the current findings as well as the prior results indicating a pro-attentional efficacy of TAK-071 (Kucinski et al. 2020) suggest that the demonstration of efficacy of TAK-071 is a function of the demands on attentional control of complex movements. It would appear less likely that this treatment benefits primarily motor parameters of gait and balance. Dual task testing situations, which have often been employed to reveal gait and balance disorders and fall risks in aged persons and PD patients (e.g., Yogev-Seligmann et al. 2008, 2012, 2013; Springer et al. 2006; Mersmann et al. 2013; Woollacott and Shumway-Cook 2002; Shumway-Cook et al. 1997; Plotnik et al. 2011), or other manipulations which strongly tax the attentional control of gait, may be crucial for demonstrating pharmacological efficacy. Finally, the present findings suggest that repeated behavioral testing in the presence of the treatment may reveal the progressively beneficial modulation of cognitive-motor interactions by this M1 PAM.

Acknowledgements We thank Dr. Arthur Simen (Takeda, Cambridge, $\mathrm{MA}$ ) for comments on a draft of this manuscript.

Author contribution AK conducted the experiments and analyzed the data. AK and MS wrote the paper.

\section{Declarations}

The research described in this manuscript was supported by NIH grant P50NS091856 (Morris K. Udall Center for Excellence in Parkinson's Disease Research). TAK-071 was obtained from Takeda Pharmaceutical Company. Dr. Sarter previously received compensation as a consultant for Takeda (2017-2019). No such consulting activity took place during the period this research was conducted (2020).

Conflict of interest The authors declare no competing interests.

\section{References}

Albin RL, Surmeier DJ, Tubert C, Sarter M, Muller M, Bohnen NI, Dauer WT (2018a) Targeting the pedunculopontine nucleus in Parkinson's disease: time to go back to the drawing board. Mov Disord 33:18711875

Albin RL, Bohnen NI, Muller M, Dauer WT, Sarter M, Frey KA, Koeppe RA (2018b) Regional vesicular acetylcholine transporter distribution in human brain: a [(18) F]fluoroethoxybenzovesamicol positron emission tomography study. J Comp Neurol 526:2884-2897

Allcock LM, Rowan EN, Steen IN, Wesnes K, Kenny RA, Burn DJ (2009) Impaired attention predicts falling in Parkinson's disease. Parkinsonism Relat Disord 15:110-115
Amboni M, Barone P, Hausdorff JM (2013) Cognitive contributions to gait and falls: evidence and implications. Mov Disord 28:1520 1533

Avila C, Kucinski A, Sarter M (2020) Complex movement control in a rat model of Parkinsonian falls: bidirectional control by striatal cholinergic interneurons. J Neurosci 40:6049-6067

Berry AS, Demeter E, Sabhapathy S, English BA, Blakely RD, Sarter M, Lustig C (2014) Disposed to distraction: genetic variation in the cholinergic system influences distractibility but not time-on-task effects. J Cogn Neurosci 26:1981-1991

Berry AS, Sarter M, Lustig C (2017) Distinct frontoparietal networks underlying attentional effort and cognitive control. J Cogn Neurosci 29:1212-1225

Bohnen NI, Albin RL (2011) The cholinergic system and Parkinson disease. Behav Brain Res 221:564-573

Bohnen NI, Müller MLTM, Koeppe RA, Studenski SA, Kilbourn MA, Frey KA, Albin RL (2009) History of falls in Parkinson disease is associated with reduced cholinergic activity. Neurology 73:1670 1676

Bohnen NI, Kanel P, Zhou Z, Koeppe RA, Frey KA, Dauer WT, Albin RL, Muller M (2019) Cholinergic system changes of falls and freezing of gait in Parkinson's disease. Ann Neurol 85:538-549

Breese GR, Traylor TD (1971) Depletion of brain noradrenaline and dopamine by 6-hydroxydopamine. Br J Pharmacol 42:88-99

Chen HC, Schultz AB, Ashton-Miller JA, Giordani B, Alexander NB, Guire KE (1996) Stepping over obstacles: dividing attention impairs performance of old more than young adults. J Gerontol A Biol Sci Med Sci 51:M116-M122

Cheng FY, Yang YR, Wang CJ, Wu YR, Cheng SJ, Wang HC, Wang RY (2014) Factors influencing turning and its relationship with falls in individuals with Parkinson's disease. PLoS One 9:e93572

Coelho FGM, Stella F, de Andrade LP, Barbieri FA, Santos-Galduróz RF, Gobbi S, Costa JLR, Gobbi LTB (2012) Gait and risk of falls associated with frontal cognitive functions at different stages of Alzheimer's disease. Neuropsychol Dev Cogn B Aging Neuropsychol Cogn 19:644-656

Cohen J (1988) Statistical power analysis for the behavioral sciences, 2nd edn. L. Erlbaum Associates, Hillsdale

Cole MH, Silburn PA, Wood JM, Kerr GK (2011) Falls in Parkinson's disease: evidence for altered stepping strategies on compliant surfaces. Parkinsonism Relat Disord 17:610-616

Cools R, Rogers R, Barker RA, Robbins TW (2010) Top-down attentional control in Parkinson's disease: salient considerations. J Cogn Neurosci 22:848-859

Disney AA, Aoki C (2008) Muscarinic acetylcholine receptors in macaque V1 are most frequently expressed by parvalbuminimmunoreactive neurons. J Comp Neurol 507:1748-1762

Fasano A, Plotnik M, Bove F, Berardelli A (2012) The neurobiology of falls. Neurol Sci 33:1215-1223

Gould RW, Dencker D, Grannan M, Bubser M, Zhan X, Wess J, Xiang Z, Locuson C, Lindsley CW, Conn PJ, Jones CK (2015) Role for the M1 muscarinic acetylcholine receptor in top-down cognitive processing using a touchscreen visual discrimination task in mice. ACS Chem Neurosci 6:1683-1695

Greenwald AG, Gonzalez R, Harris RJ, Guthrie D (1996) Effect sizes and p values: what should be reported and what should be replicated? Psychophysiology 33:175-183

Grimbergen YA, Munneke M, Bloem BR (2004) Falls in Parkinson's disease. Curr Opin Neurol 17:405-415

Gritton HJ, Howe WM, Mallory CS, Hetrick VL, Berke JD, Sarter M (2016) Cortical cholinergic signaling controls the detection of cues. Proc Natl Acad Sci U S A 113:E1089-E1097

Heremans E, Nieuwboer A, Vercruysse S (2013) Freezing of gait in Parkinson's disease: where are we now? Curr Neurol Neurosci Rep 13:350-359 
Herrero JL, Roberts MJ, Delicato LS, Gieselmann MA, Dayan P, Thiele A (2008) Acetylcholine contributes through muscarinic receptors to attentional modulation in V1. Nature 454:1110-1114

Hollman JH, Youdas JW, Lanzino DJ (2011) Gender differences in dual task gait performance in older adults. Am J Mens Health 5:11-17

Holtzer R, Wang C, Verghese J (2014) Performance variance on walking while talking tasks: theory, findings, and clinical implications. Age (Dordr) 36:373-381

Howe WM, Berry AS, Francois J, Gilmour G, Carp JM, Tricklebank M, Lustig C, Sarter M (2013) Prefrontal cholinergic mechanisms instigating shifts from monitoring for cues to cue-guided performance: converging electrochemical and fMRI evidence from rats and humans. J Neurosci 33:8742-8752

Howe WM, Gritton HJ, Lusk NA, Roberts EA, Hetrick VL, Berke JD, Sarter M (2017) Acetylcholine release in prefrontal cortex promotes gamma oscillations and theta-gamma coupling during cue detection. J Neurosci 37:3215-3230

Kehagia AA, Barker RA, Robbins TW (2013) Cognitive impairment in Parkinson's disease: the dual syndrome hypothesis. Neurodegener Dis 11:79-92

Kim K, Muller M, Bohnen NI, Sarter M, Lustig C (2017) Thalamic cholinergic innervation makes a specific bottom-up contribution to signal detection: evidence from Parkinson's disease patients with defined cholinergic losses. Neuroimage 149:295-304

Kim K, Muller M, Bohnen NI, Sarter M, Lustig C (2019) The cortical cholinergic system contributes to the top-down control of distraction: evidence from patients with Parkinson's disease. Neuroimage 190:107-117

Koshy Cherian A, Kucinski A, Wu R, de Jong IEM, Sarter M (2019) Cotreatment with rivastigmine and idalopirdine reduces the propensity for falls in a rat model of falls in Parkinson's disease. Psychopharmacology 236:1701-1715

Kucinski A, Sarter M (2015) Modeling Parkinson's disease falls associated with brainstem cholinergic systems decline. Behav Neurosci 129:96-104

Kucinski A, Sarter M (2016) Cortico-striatal-cognitive-motor interactions underlying complex movement control deficits. In: Soghomonian JJ (ed) The basal ganglia-novel perspectives on motor and cognitive functions. Springer International Publishing, pp 117-134

Kucinski A, Paolone G, Bradshaw M, Albin R, Sarter M (2013) Modeling fall propensity in Parkinson's disease: deficits in the attentional control of complex movements in rats with corticalcholinergic and striatal-dopaminergic deafferentation. J Neurosci 33:16522-16539

Kucinski A, Albin RL, Lustig C, Sarter M (2015) Modeling falls in Parkinson's disease: slow gait, freezing episodes and falls in rats with extensive striatal dopamine loss. Behav Brain Res 282:155164

Kucinski A, de Jong IE, Sarter M (2017) Reducing falls in Parkinson's disease: interactions between donepezil and the 5-HT6 receptor antagonist idalopirdine on falls in a rat model of impaired cognitive control of complex movements. Eur J Neurosci 45:217-231

Kucinski A, Lustig C, Sarter M (2018) Addiction vulnerability trait impacts complex movement control: evidence from sign-trackers. Behav Brain Res 350:139-148

Kucinski A, Kim Y, Sarter M (2019) Basal forebrain chemogenetic inhibition disrupts the superior complex movement control of goaltracking rats. Behav Neurosci 133:121-134

Kucinski A, Phillips KB, Koshy Cherian A, Sarter M (2020) Rescuing the attentional performance of rats with cholinergic losses by the M1 positive allosteric modulator TAK-071. Psychopharmacology 237: $137-153$

Kurimoto E, Nakashima M, Kimura H, Suzuki M (2019) TAK-071, a muscarinic M1 receptor positive allosteric modulator, attenuates scopolamine-induced quantitative electroencephalogram power spectral changes in cynomolgus monkeys. PLoS One 14:e0207969
Langston JW (2006) The Parkinson's complex: Parkinsonism is just the tip of the iceberg. Ann Neurol 59:591-596

Li Z, Yu Z, Zhang J, Wang J, Sun C, Wang P, Zhang J (2015) Impact of rivastigmine on cognitive dysfunction and falling in Parkinson's disease patients. Eur Neurol 74:86-91

Lissek S, Hausmann M, Knossalla F, Peters S, Nicolas V, Gunturkun O, Tegenthoff $M$ (2007) Sex differences in cortical and subcortical recruitment during simple and complex motor control: an fMRI study. Neuroimage 37:912-926

Lord S, Rochester L, Hetherington V, Allcock LM, Burn D (2010) Executive dysfunction and attention contribute to gait interference in 'off' state Parkinson's disease. Gait Posture 31:169-174

Lu Y, Sarter M, Zochowski M, Booth V (2020) Phasic cholinergic signaling promotes emergence of local gamma rhythms in excitatoryinhibitory networks. Eur J Neurosci 52:3545-3560

Mersmann F, Bohm S, Bierbaum S, Dietrich R, Arampatzis A (2013) Young and old adults prioritize dynamic stability control following gait perturbations when performing a concurrent cognitive task. Gait Posture 37:373-377

Montero-Odasso M, Verghese J, Beauchet O, Hausdorff JM (2012) Gait and cognition: a complementary approach to understanding brain function and the risk of falling. J Am Geriatr Soc 60:2127-2136

Moran SP, Maksymetz J, Conn PJ (2019) Targeting muscarinic acetylcholine receptors for the treatment of psychiatric and neurological disorders. Trends Pharmacol Sci 40:1006-1020

Muller ML, Albin RL, Kotagal V, Koeppe RA, Scott PJ, Frey KA, Bohnen NI (2013) Thalamic cholinergic innervation and postural sensory integration function in Parkinson's disease. Brain 136: 3282-3289

Parikh V, Kozak R, Martinez V, Sarter M (2007) Prefrontal acetylcholine release controls cue detection on multiple timescales. Neuron 56 : $141-154$

Paul SS, Sherrington C, Canning CG, Fung VS, Close JC, Lord SR (2014) The relative contribution of physical and cognitive fall risk factors in people with Parkinson's disease: a large prospective cohort study. Neurorehabil Neural Repair 28:282-290

Pereira CL, Baptista F, Infante P (2013) Men older than 50 yrs are more likely to fall than women under similar conditions of health, body composition, and balance. Am J Phys Med Rehabil 92:1095-1103

Picciotto MR, Higley Michael J, Mineur Yann S (2012) Acetylcholine as a neuromodulator: cholinergic signaling shapes nervous system function and behavior. Neuron 76:116-129

Plotnik M, Giladi N, Dagan Y, Hausdorff JM (2011) Postural instability and fall risk in Parkinson's disease: impaired dual tasking, pacing, and bilateral coordination of gait during the "ON" medication state. Exp Brain Res 210:529-538

Rochester L, Yarnall AJ, Baker MR, David RV, Lord S, Galna B, Burn DJ (2012) Cholinergic dysfunction contributes to gait disturbance in early Parkinson's disease. Brain 135:2779-2788

Sako Y, Kurimoto E, Mandai T, Suzuki A, Tanaka M, Suzuki M, Shimizu Y, Yamada M, Kimura H (2018) TAK-071, a novel M1 positive allosteric modulator with low cooperativity, improves cognitive function in rodents with few cholinergic side effects. Neuropsychopharmacology.

Sarter M (2006) Preclinical research into cognition enhancers. Trends Pharmacol Sci 27:602-608

Sarter M, Fritschy JM (2008) Reporting statistical methods and statistical results in EJN. Eur J Neurosci 28:2363-2364

Sarter M, Lustig C (2020) Forebrain cholinergic signaling: wired and phasic, not tonic, and causing behavior. J Neurosci 40:712-719

Sarter M, Albin RL, Kucinski A, Lustig C (2014) Where attention falls: increased risk of falls from the converging impact of cortical cholinergic and midbrain dopamine loss on striatal function. Exp Neurol $257: 120-129$ 
Shine JM, Moustafa AA, Matar E, Frank MJ, Lewis SJ (2013) The role of frontostriatal impairment in freezing of gait in Parkinson's disease. Front Syst Neurosci 7:61

Shumway-Cook A, Woollacott M (2000) Attentional demands and postural control: the effect of sensory context. J Gerontol A Biol Sci Med Sci 55:M10-M16

Shumway-Cook A, Woollacott M, Kerns KA, Baldwin M (1997) The effects of two types of cognitive tasks on postural stability in older adults with and without a history of falls. J Gerontol A Biol Sci Med Sci 52:M232-M240

Springer S, Giladi N, Peretz C, Yogev G, Simon ES, Hausdorff JM (2006) Dual-tasking effects on gait variability: the role of aging, falls, and executive function. Mov Disord 21:950-957

Stack E, Ashburn A (2008) Dysfunctional turning in Parkinson's disease. Disabil Rehabil 30:1222-1229

Stam CJ, Visser SL, Op de Coul AA, De Sonneville LM, Schellens RL, Brunia CH, de Smet JS, Gielen G (1993) Disturbed frontal regulation of attention in Parkinson's disease. Brain 116(Pt 5):1139-1158

Stuart S, Galna B, Delicato LS, Lord S, Rochester L (2017) Direct and indirect effects of attention and visual function on gait impairment in Parkinson's disease: influence of task and turning. Eur J Neurosci 46:1703-1716

Taylor TN, Caudle WM, Shepherd KR, Noorian A, Jackson CR, Iuvone PM, Weinshenker D, Greene JG, Miller GW (2009) Nonmotor symptoms of Parkinson's disease revealed in an animal model with reduced monoamine storage capacity. J Neurosci 29:8103-8113
Venkatesan S, Jeoung HS, Chen T, Power SK, Liu Y, Lambe EK (2020) Endogenous acetylcholine and its modulation of cortical microcircuits to enhance cognition. Curr Top Behav Neurosci 45:47-69

Weaver TB, Robinovitch SN, Laing AC, Yang Y (2016) Falls and Parkinson's disease: evidence from video recordings of actual fall events. J Am Geriatr Soc 64:96-101

Wong AL, Haith AM, Krakauer JW (2015) Motor planning. Neuroscientist 21:385-398

Woollacott M, Shumway-Cook A (2002) Attention and the control of posture and gait: a review of an emerging area of research. Gait Posture 16:1-14

Yarnall A, Rochester L, Burn DJ (2011) The interplay of cholinergic function, attention, and falls in Parkinson's disease. Mov Disord 26:2496-2503

Yogev-Seligmann G, Hausdorff JM, Giladi N (2008) The role of executive function and attention in gait. Mov Disord 23:329-342

Yogev-Seligmann G, Hausdorff JM, Giladi N (2012) Do we always prioritize balance when walking? Towards an integrated model of task prioritization. Mov Disord 27:765-770

Yogev-Seligmann G, Giladi N, Gruendlinger L, Hausdorff JM (2013) The contribution of postural control and bilateral coordination to the impact of dual tasking on gait. Exp Brain Res 226:81-93

Publisher's note Springer Nature remains neutral with regard to jurisdictional claims in published maps and institutional affiliations. 